Recepción: 20 / 04 / 2017

Aceptación: 20 / 05 / 2017

Publicación: 15 / 06 / 2017

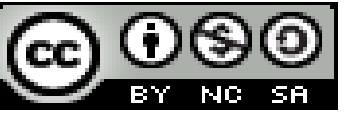

\title{
Estudio de las concentraciones por metales pesados e hidrocarburos en el ramal B del Estero Salado
}

Study of concentrations by heavy metals and hydrocarbons in branch B of Estero

\section{Salado}

As concentrações de estudo de metais pesados e hidrocarbonetos no ramo B do Sal Matting

\author{
Judith A. Chalen-Medina ${ }^{\mathrm{I}}$ \\ judith.chalem@ug.edu.ec \\ Gino F. Flor-Chávez II \\ ginoflorch@ug.edu.ec \\ Kerly C. Fun- Sang III \\ kerly.funsangr@ug.edu.ec
}

Correspondencia: judith.chalem@ug.edu.ec

\footnotetext{
I Ingeniera Química; Magister en Ingeniería Ambiental; Universidad de Guayaquil, Guayaquil, Ecuador. Ingeniero Civil; Magister en Ingeniería Vial; Universidad de Guayaquil, Guayaquil, Ecuador.

Arquitectura, Magister en Impactos Ambientales; Docente Universidad de Guayaquil, Guayaquil, Ecuador.
} 


\section{Resumen}

El Estero Salado es un sistema estuarino ubicado en la ciudad de Guayaquil, además es considerado uno de los medios acuáticos importantes de nuestro país, en las últimas décadas la presencia de contaminantes peligrosos ha dado como resultado un problema de degradación tanto en aguas como en suelo perdiendo así su belleza natural. Es por eso que el objetivo general de este estudio fue determinar la presencia de metales pesados e hidrocarburos en el ramal B del Estero Salado tanto en aguas como en sedimento marino. Se escogió este tramo debido a que es una zona muy afectada por el sector industrial y urbanístico.

La metodología aplicada fue la recolección de muestra sólidas como líquidas en seis estaciones establecidas en el tramo señalado según el GPS, dichas muestras fueron analizadas en laboratorio realizándose análisis de: contaminantes (arsénico, cadmio, plomo, mercurio, hidrocarburos totales de petróleo y fenoles), físico-químico (sólidos totales, potencial hidrógeno) y materia biodegradable (expresada como: demanda bioquímica de oxígeno y demanda química de oxígeno). Los resultados demuestran que los contaminantes señalados (excepto arsénico), sólidos totales y demanda bioquímica de oxígeno, están al borde y superan el límite máximo permisible establecidos por las legislaciones ambientales vigentes, en ambos estados, lo que nos da a entender que este ramal posee un alto contenido de metales pesados y compuestos hidrocarburíferos, dando como consecuencia el poco contenido de oxígeno y degradación en sus aguas.

Palabras claves: Metales pesados, compuestos hidrocarburíferos, físico-químico, bioquímico 


\begin{abstract}
Estero Salado is an estuarine system located in the city of Guayaquil, in addition it is considered one of the important aquatic environments of our country, in the last decades the presence of dangerous pollutants has resulted in a problem of degradation in both water and soil Thus losing its natural beauty. That is why the general objective of this study was to determine the presence of heavy metals and hydrocarbons in branch B of the Estero Salado in both waters and marine sediment. This section was chosen because it is an area very affected by the industrial and urban sector.

The methodology used was the collection of solid samples as liquids in six stations established in the designated section according to the GPS. These samples were analyzed in the laboratory by analyzing: contaminants (arsenic, cadmium, lead, mercury, total petroleum hydrocarbons and phenols), Physical-chemical (total solids, hydrogen potential) and biodegradable matter (expressed as: biochemical oxygen demand and chemical oxygen demand). The results show that the indicated pollutants (except arsenic), total solids and biochemical oxygen demand, are on the edge and exceed the maximum permissible limit established by the current environmental legislation in both states, which suggests that this branch possesses A high content of heavy metals and hydrocarbon compounds, resulting in low oxygen content and degradation in their waters
\end{abstract}

Key words: Heavy metals, hydrocarbon compounds, physicochemical, biochemical 


\section{Resumo}

O Estero Salado é um sistema estuarino localizado na cidade de Guayaquil, também é considerado um dos principais recursos hídricos do nosso país, nas últimas décadas, a presença de poluentes perigosos resultou em um problema de degradação em água e solo perdendo assim a sua beleza natural. É por isso que o objetivo geral deste estudo foi determinar a presença de metais pesados e hidrocarbonetos no ramo B do Sal Matting água e sedimentos marinhos. este trecho porque é uma área muito afetada pelo setor industrial e urbana foi escolhida.

A metodologia foi a recolha de amostra de sólido e líquido em seis estações estabelecidos na secção identificada como o GPS, as amostras foram analisadas no laboratório que realiza análise: contaminantes (arsénio, cádmio, chumbo, mercúrio, hidrocarbonetos de petróleo e fenóis), físicoquímica (sólidos totais, hidrogénio potencial) e material biodegradável (expressa como demanda bioquímica de oxigénio e a demanda química de oxigénio). Os resultados demonstram que os contaminantes enumerados (excepto o arsénio), sólidos totais e demanda bioquímica de oxigénio, estão a ponto e exceder o limite máximo estabelecido pela legislação ambiental em vigor, em ambos os estados, o que sugere que este ramo tem um elevado teor de metais pesados e de compostos de hidrocarbonetos, dando como resultado o pequeno teor de oxigénio e degradação na sua água.

Palavras-chave: metais pesados, hidrocarbonetos, compostos de características físico-químicas, bioquímicas 


\section{Introducción.}

El Estero Salado es un recurso estuarino situado en el sector nor - oeste y sur - este de la ciudad de Guayaquil, realizando su desembocadura en el golfo de la misma ciudad, abarcando una distancia de aproximadamente $90 \mathrm{~km}$. Es un brazo de mar; el cual junto con las partes bajas del río Daule y Babahoyo forman parte del ecosistema denominado Estuario Interior del Golfo de Guayaquil (Cárdenas, 2010). El Estero Salado, ha sido uno de los elementos que identifican a la ciudad y al guayaquileño, por décadas ícono natural y termómetro ambiental de la ciudad. Con su vegetación, compuesta por $90 \%$ de mangle, regula el clima de Guayaquil y constituye un paraíso de algunas especies como: conchas, cangrejos, mejillones y variedades de peces (Cervantes, 2012)

\section{Materiales y métodos.}

\section{Métodos teóricos}

\section{Selección de la zona de estudio}

El ramal B del Estero Salado fue seleccionado para realizar esta investigación debido a que es considerada una zona afectada por el sector industrial (ubicada en sus alrededores) debido a la descarga de sus aguas residuales. Otro problema de contaminación que tiene este sector son las aguas residuales de uso doméstico provenientes de las urbanizaciones aledañas al sector (MIDUVI - MAE, 2013).

\section{Descripción de la zona de estudio}

Este tramo está ubicado en el norte de la ciudad, está formada por áreas habitadas con servicios urbanísticos, calles asfaltadas, pasos a desnivel, servicios de agua potable, 
alcantarillado, entre otros. Esta zona se inicia desde el Complejo Polideportivo de la ciudadela Miraflores, pasando por las urbanizaciones: Miraflores, Quisquis y Urdesa Central, terminando en el puente Zig - Zag (punto de unión entre las universidades: Guayaquil y Católica). Este trayecto mide aproximadamente $2 \mathrm{~km}$ de distancia y en todo este sector se puede observar que este recurso estuarino tiene una menor influencia de mareas y pequeñas áreas de bosque interrumpidas por urbanizaciones.

\section{Ubicación de las estaciones}

Las estaciones de muestreo fueron localizadas por medio del Sistema de Posicionamiento Global (GPS), en la cual se detectaron 6 estaciones con una distancia de 0,25 a 0,60 km. En la tabla 4 y figura 5 se muestran las ubicaciones de cada una de las estaciones de muestreo.

\begin{tabular}{ccc}
\hline Estación & Zona & Coordenadas \\
\hline 1 & Complejo polideportivo Miraflores & $61998-9761028$ \\
2 & Avenida Miraflores & $620190-9760925$ \\
3 & Av. Dr. Ignacio Cuesta Garcés & $620392-9760854$ \\
4 & Centro Comercial Albán Borja & $620471-9760439$ \\
5 & Avenida Las Monjas & $620883-9759822$ \\
6 & Puente Zic - Zac & $621950-9759224$ \\
\hline & Tabla 1 Estaciones identificadas en el tramo de estudio \\
& Fuente: Sistema de Posicionamiento Global (GPS)
\end{tabular}

\section{Métodos Empíricos}

Una vez ingresadas las muestras se procedió a la distribución para su análisis en el Grupo Químico Marcos. En la tabla 5 se muestra los métodos aplicados a cada uno de ellos, basados en el método analítico (STANDARD METHODS, 22 TH EDITION, 2012). Cabe indicar que el 
Grupo Químico Marcos es un laboratorio ambiental de análisis acreditado por la norma ISO 17025. Se trata de una norma de calidad, que deben de cumplir los laboratorios de ensayos en producir resultados confiables. La acreditación esta registra por el Servicio de Acreditación Ecuatoriana (SAE) con acreditación No OAE LE 2C 05-001.

\begin{tabular}{cc}
\hline Parámetros & Método analítico \\
\hline Metales pesados en sedimento & PEE - GQM - FQ - 54 \\
Metales pesados en líquido & PEE - GQM - FQ - 33 \\
TPH en sedimento & PEE - GQM - FQ - 56 \\
TPH en líquido & PEE - GQM - FQ - 07 \\
Fenoles & PEE - GQM - FQ - 20 \\
Sólidos suspendidos & PEE - GQM - FQ - 06 \\
Sólidos disueltos & PEE - GQM - FQ - 41 \\
pH & PEE - GQM - FQ - 41 \\
DBO5 & PEE - GQM - FQ - 17 \\
DQO & PEE - GQM - FQ - 16 \\
Tabla 2 Métodos aplicados en los parámetros estudiados \\
Fuente: www.grupoquimicomarcos.com
\end{tabular}

\section{Resultados.}

Resultados de los análisis en muestras líquidas

Metales pesados

En la tabla 3 se encuentra presente el contenido de cada uno de los metales pesados en las seis estaciones correspondientes al ramal B del Estero Salado. En la figura 1 se observa que el 
arsénico (As) y el cadmio (Cd) mantienen las mismas concentraciones en la mayoría de las estaciones, donde sus contenidos están muy por debajo del límite máximo permisible.

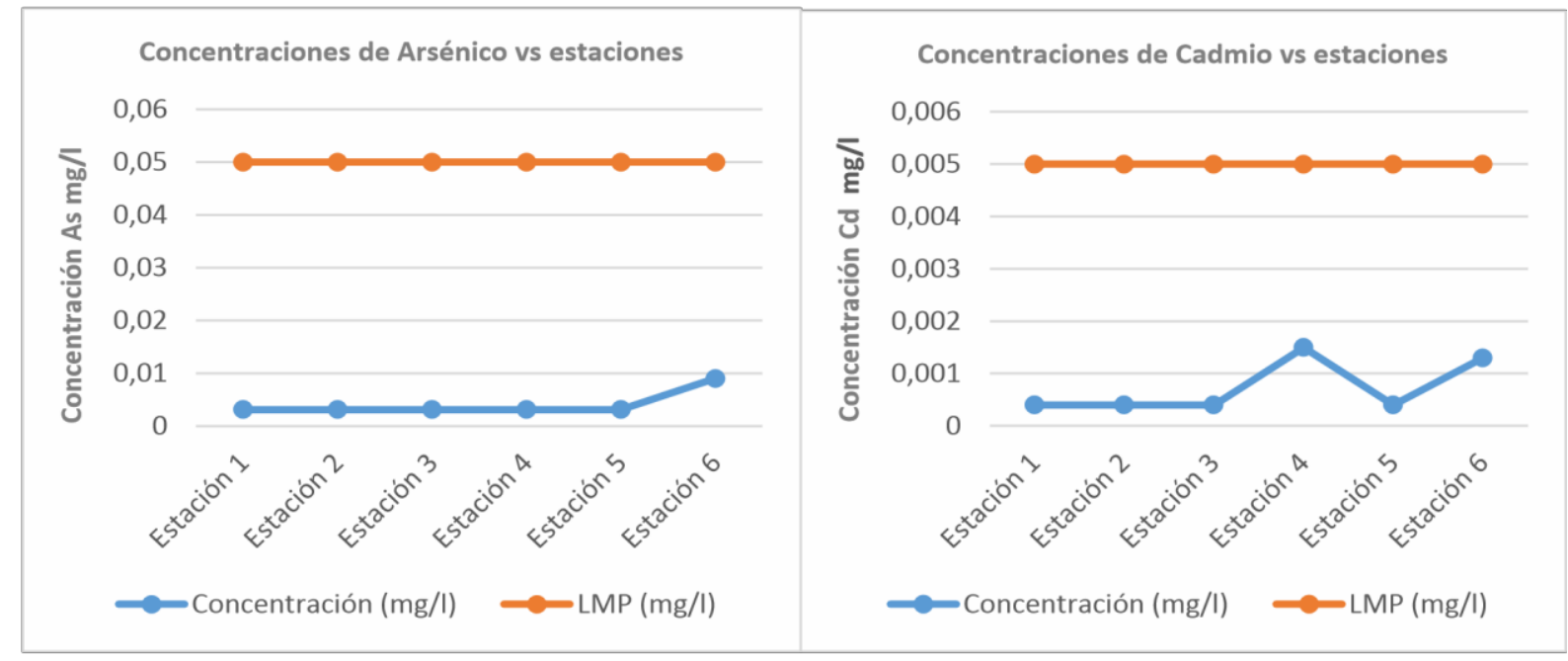

\section{Figura 1: Evoluciones de las concentraciones de As y Cd en el Ramal B Estero Salado Fuente: Autor}

Mientras tanto metales como el mercurio $(\mathrm{Hg})$ y el plomo $(\mathrm{Pb})$ sus concentraciones varían de acuerdo a las estaciones, pero sus promedios sobrepasan el límite máximo permisible, siendo este último el metal de mayor contenido en muestras líquidas. En la figura 2 se muestra el comportamiento de estos elementos en las estaciones.

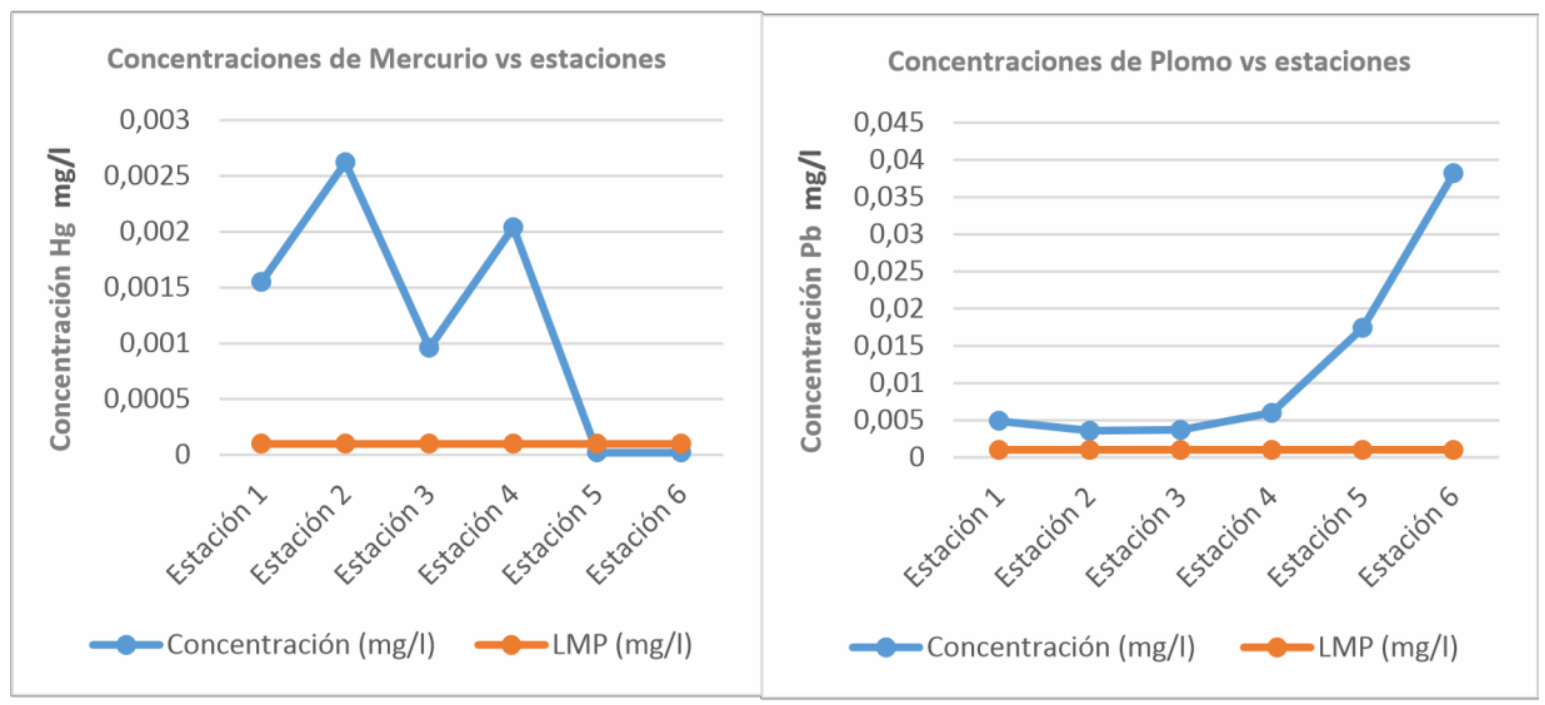


Figura 2: Evoluciones de las concentraciones de $\mathrm{Hg}$ y $\mathrm{Pb}$ en el Ramal B Estero Salado Fuente: Autor

\begin{tabular}{|c|c|c|c|c|}
\hline Estación & As (mg/l) & $\mathrm{Cd}(\mathrm{mg} / \mathrm{l})$ & $\mathrm{Hg}(\mathrm{mg} / \mathrm{l})$ & $\mathrm{Pb}(\mathrm{mg} / \mathrm{l})$ \\
\hline Estación 1 & 0,0031 & 0,0004 & 0,00155 & 0,0049 \\
\hline Estación 2 & 0,0031 & 0,0004 & 0,00262 & 0,0036 \\
\hline Estación 3 & 0,0031 & 0,0004 & 0,00096 & 0,0037 \\
\hline Estación 4 & 0,0031 & 0,0015 & 0,00204 & 0,0060 \\
\hline Estación 5 & 0,0031 & 0,0004 & 0,00002 & 0,0174 \\
\hline \multirow[t]{2}{*}{ Estación 6} & 0,0090 & 0,0013 & 0,00002 & 0,0382 \\
\hline & 0,0041 & 0,0007 & 0,0012 & 0,0123 \\
\hline $\begin{array}{l}\text { Promedio } \\
\text { Límite permitido }\end{array}$ & & & & \\
\hline (TULAS, 2015) & 0,05 & 0,005 & 0,0001 & 0,001 \\
\hline
\end{tabular}

Compuestos hidrocarburíferos

En la tabla 4 se puede ver los contenidos de los derivados del petróleo: hidrocarburos totales de petróleo (TPH) y fenoles. Los TPH presentan la misma concentración, está dentro del límite permisible pero muy cerca de su valor máximo por lo que existe una concentración media alta de este contaminante. Los fenoles en la mayoría de las estaciones poseen la misma concentración sobrepasando su límite según la norma ambiental obteniendo un promedio de $0,023 \mathrm{mg} / \mathrm{l}$. En la figura 3 se puede visualizar una gráfica de las concentraciones de los derivados del petróleo. 
Judith A. Chalen-Medina; Gino F. Flor-Chavez; Kerly C. Fun- Sang

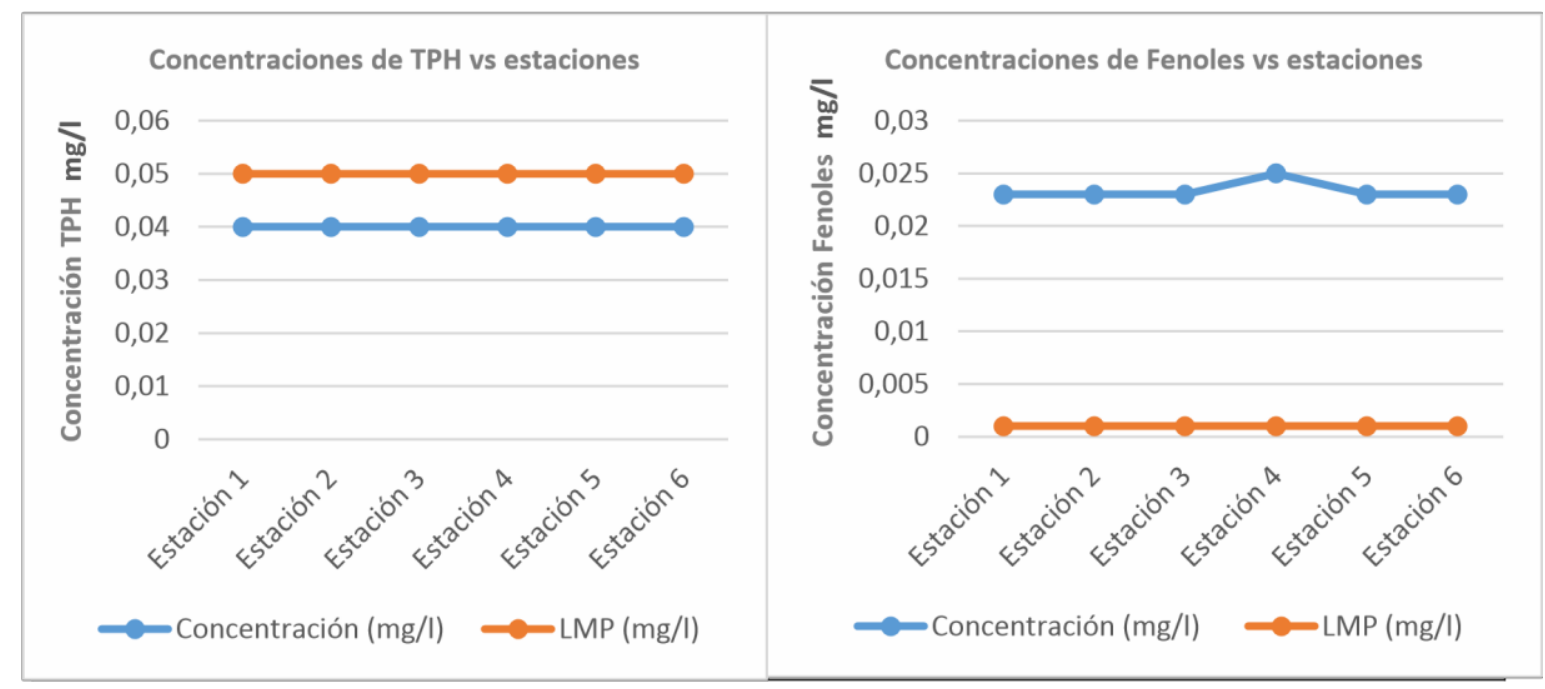

Figura 3: Evoluciones de las concentraciones de derivados del petróleo

Fuente: Autor

\begin{tabular}{ccc}
\hline Estaciones & TPH $(\mathbf{m g} / \mathbf{l})$ & Fenoles $(\mathbf{m g} / \mathbf{l})$ \\
\hline Estación 1 & 0,04 & 0,023 \\
Estación 2 & 0,04 & 0,023 \\
Estación 3 & 0,04 & 0,023 \\
Estación 4 & 0,04 & 0,025 \\
Estación 5 & 0,04 & 0,023 \\
Estación 6 & 0,04 & 0,023 \\
Promedio & 0,04 & 0,023 \\
Límite permitido (TULA, 2015) & 0,05 & 0,001
\end{tabular}

Tabla4 Contenidos de derivados de petróleo en aguas del ramal B Estero Salado Fuente: Grupo Químico Marcos

Parámetros físico químicos

En la tabla 5 se puede verificar el contenido de los parámetros físico químicos: sólidos totales y potencial hidrógeno $(\mathrm{pH})$. En el primer parámetro se puede constatar que a medida que avanzamos 
de estación las concentraciones va en ascenso, donde las dos últimas estaciones superan el límite permisible y su promedio final es casi el doble de lo permitido. En el segundo parámetro las niveles de pH están dentro del límite máximo permitido, en la figura 4 se puede apreciar una gráfica de estos parámetros físicos químicos.

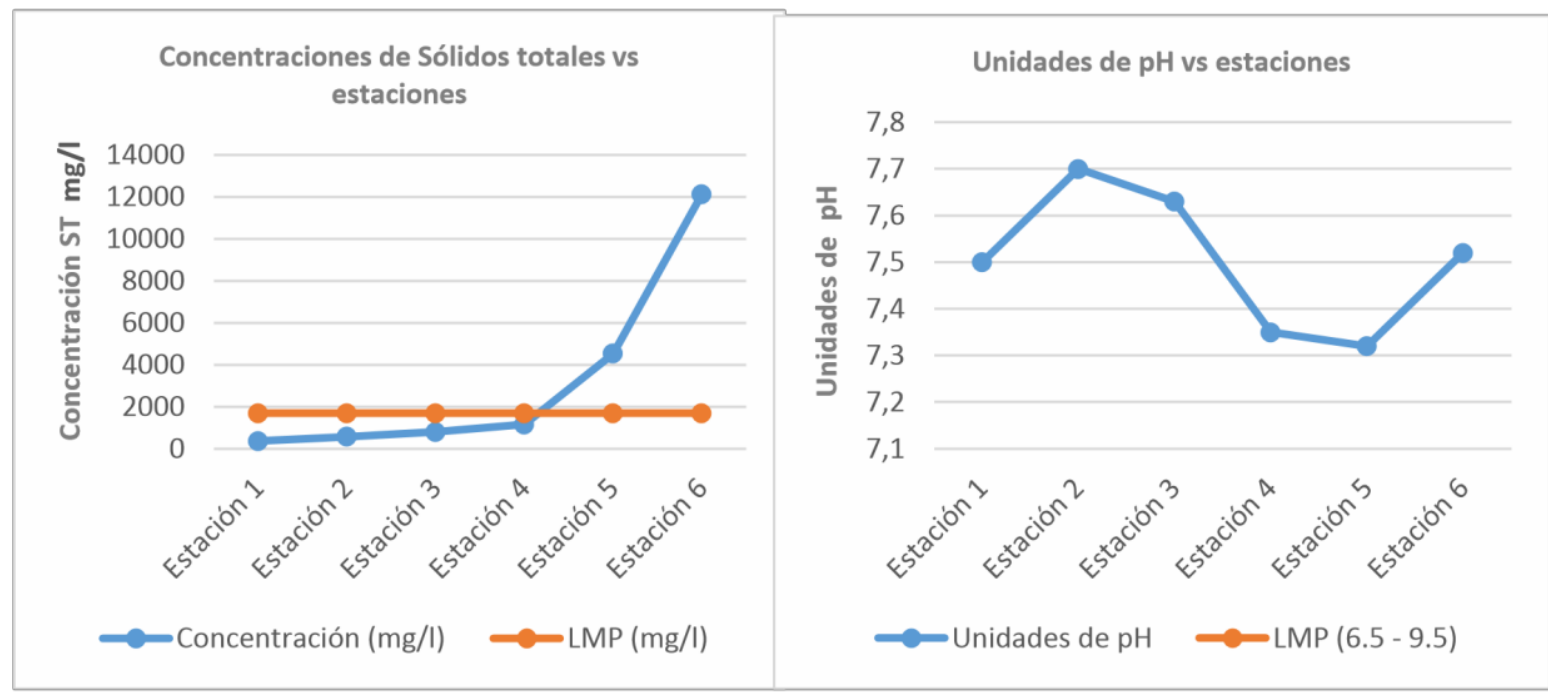

Figura 4: Evoluciones de las concentraciones de parámetros químicos Fuente: Autor

\begin{tabular}{ccc}
\hline Estaciones & Sólidos totales $(\mathbf{m g} / \mathbf{l})$ & $\mathbf{p H}$ \\
\hline Estación 1 & 374 & 7,5 \\
Estación 2 & 578 & 7,7 \\
Estación 3 & 809 & 7,63 \\
Estación 4 & 1166 & 7,63 \\
Estación 5 & 4543 & 7,35 \\
Estación 6 & 12132 & 7,32 \\
Promedio & 3267 & 7,52 \\
Límite permitido & 1700 & $6,5-9,5$ \\
& (RAOHE, 2001) & TULA, 2015) \\
\hline
\end{tabular}

Tabla 5 Contenidos de parámetros físicos químicos en aguas ramal B Estero Salado Fuente: Grupo Químico Marcos 
Determinación de la materia biodegradable expresada como $\mathrm{DQO}$ y $\mathrm{DBO}_{5}$

En la tabla 6 se presenta la demanda bioquímica de oxígeno (DBO) y demanda química de oxígeno (DQO). En la DBO se puede observar que este parámetro en el ramal B de Estero Salado tiene una concentración baja, media y alta, las tres primeras estaciones están cerca del límite máximo, las dos últimas están lejos del límite permitido y la cuarta estación es la única que supera los valores establecidos. En la DQO sus concentraciones están muy por debajo del límite máximo permisible, debido a la degradación de la materia orgánica en la figura 5 se ilustra una gráfica de estos contenidos.

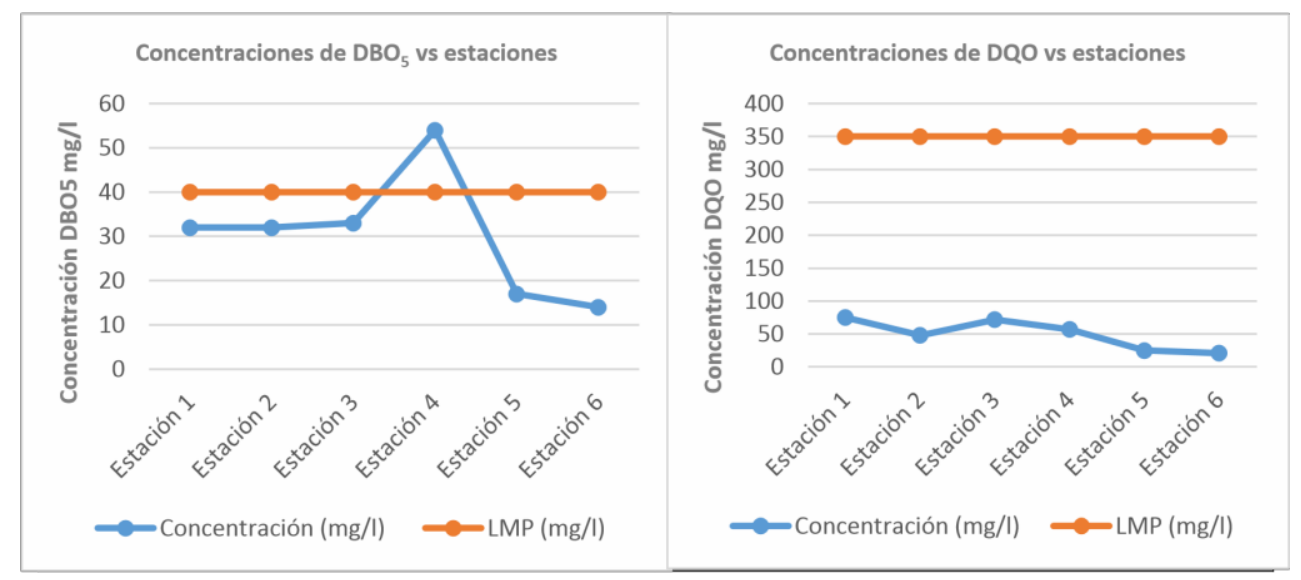

Figura 5: Evoluciones de las concentraciones de materia biodegradable Fuente: Autor 


\begin{tabular}{ccc}
\hline Estaciones & DBO (mg/l) & DQO (mg/l) \\
\hline Estación 1 & 32 & 75 \\
Estación 2 & 32 & 48 \\
Estación 3 & 33 & 72 \\
Estación 4 & 54 & 57 \\
Estación 5 & 17 & 25 \\
Estación 6 & 14 & 21 \\
Promedio & 30,33 & 49,67 \\
Límite permitido (RAOHE,2001) & 40 & 350 \\
\hline Tabla 6 Contenidos de materia biodegradable en aguas del ramal B Estero Salado
\end{tabular}

Resultados de los análisis en muestras sedimentarias

Concentración de metales pesados

En la tabla 7 se puede observar la concentración de los metales pesados estudiados en cada uno de los sedimentos de las estaciones del ramal B del estero salado, la cual se puede observar que el Cadmio y Mercurio sus concentraciones superan el límite máximo permisible en cada uno de los sectores planteados en este estudio (Figura 6). 
Judith A. Chalen-Medina; Gino F. Flor-Chavez; Kerly C. Fun- Sang

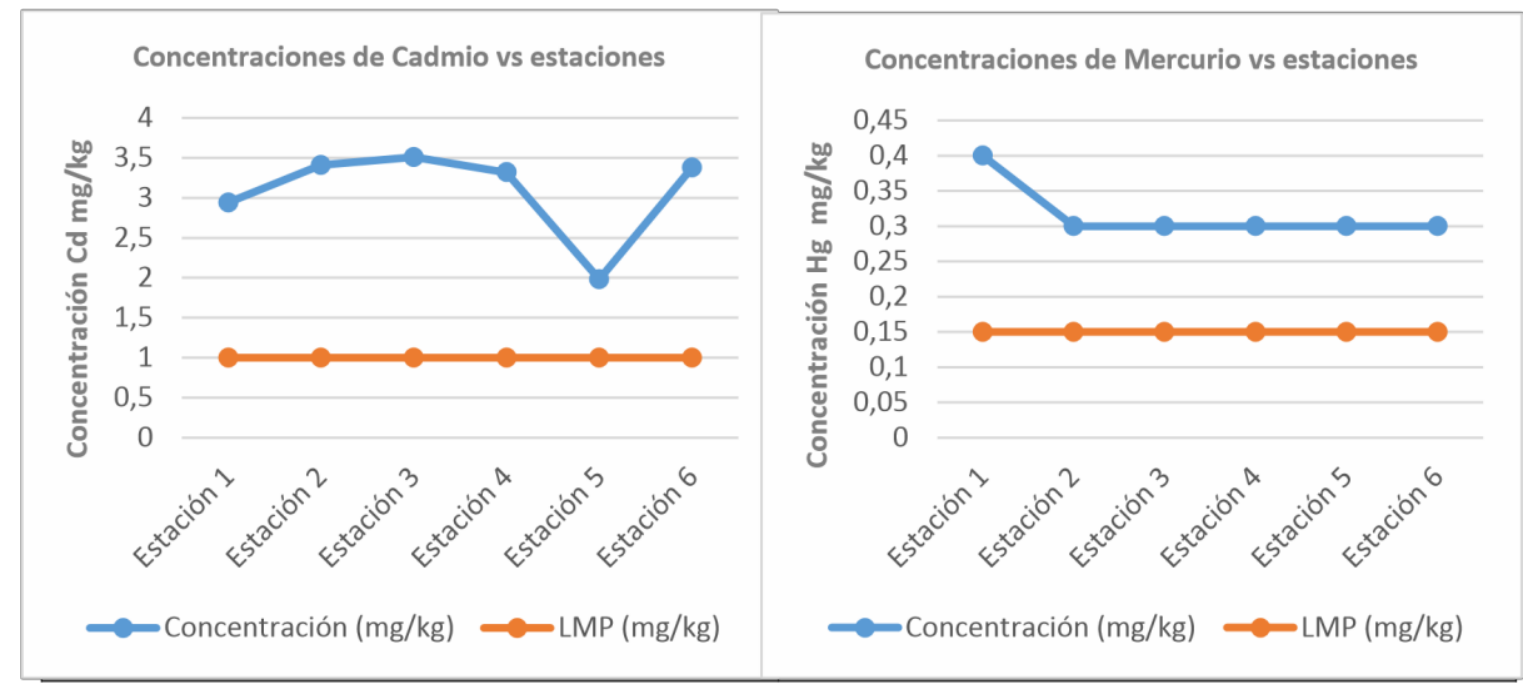

Figura 6: Evoluciones de los contenidos de cadmio y mercurio en sedimiento marino Fuente: Autor

El Arsénico se encuentra dentro del límite permisible debido que sus concentraciones no superan el $\mathrm{mg} / \mathrm{kg}$. El plomo se observa que existe una variación entre sus contenidos, en la figura 7 se muestra una gráfica de las concentraciones de estos dos elementos, donde el plomo (tomando como referencia la tabla 7 y su variación) es el metal con la mayor concentración en prototipos sedimentarios.

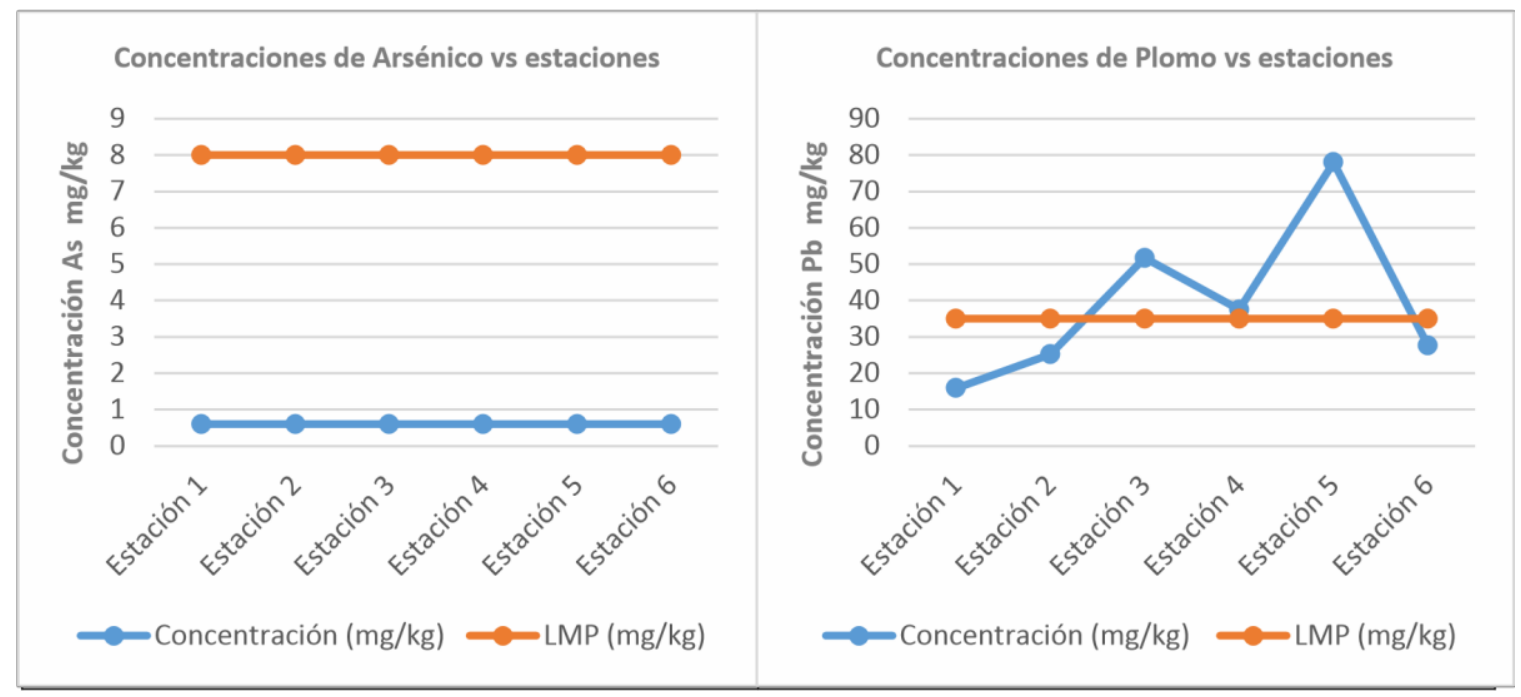

Figura 6: Evoluciones de los contenidos de arsénico y plomo en sedimiento marino Fuente: Autor 


\begin{tabular}{ccccc}
\hline Estación & $\mathbf{C d}(\mathbf{m g} / \mathbf{k g})$ & $\mathbf{H g}(\mathbf{m g} / \mathbf{k g})$ & $\mathbf{A s}(\mathbf{m g} / \mathbf{k g})$ & $\mathbf{P b}(\mathbf{m g} / \mathbf{k g})$ \\
\hline Estación 1 & 2,94 & 0,4 & 0,6 & 15,9 \\
Estación 2 & 3,41 & 0,3 & 0,6 & 25,2 \\
Estación 3 & 3,51 & 0,3 & 0,6 & 51,7 \\
Estación 4 & 3,32 & 0,3 & 0,6 & 37,5 \\
Estación 5 & 1,98 & 0,3 & 0,6 & 78 \\
Estación 6 & 3,38 & 0,3 & 0,6 & 27,6 \\
Promedio & 3,09 & 0,32 & 0,6 & 39,32 \\
Límite permitido & 1 & 0,15 & 8 & 35 \\
(Norma mundial) & \multicolumn{5}{c}{ Fuente: Grupo Químico Marcos } \\
\hline Tabla 7 Contenidos de metales pesados en sedimento del ramal B Estero Salado \\
\multicolumn{5}{c}{}
\end{tabular}

Concentración de Hidrocarburos totales de petróleo

En la tabla 8 se presentan los contenidos de TPH (Hidrocarburos totales de petróleo) en las estaciones muestreadas, donde sus valores están al borde del límite máximo permisible, es decir, existe una concentración media alta de estos derivados del petróleo en los sedimentos del ramal B del Estero Salado. En la figura 8 se presenta una grafica 
Judith A. Chalen-Medina; Gino F. Flor-Chavez; Kerly C. Fun- Sang

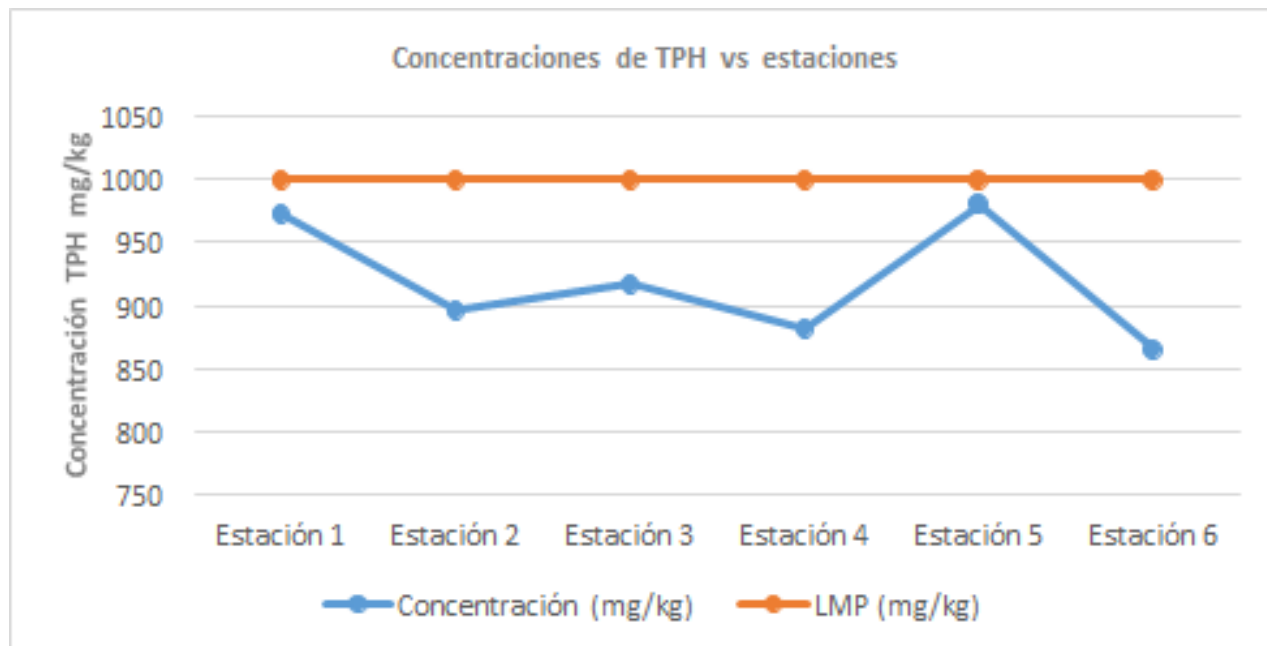

Figura 8: Evoluciones de los contenidos de TPH en sedimiento marino

Fuente: Autor

\begin{tabular}{cc}
\hline Estación & Hidrocarburos totales de petróleo (mg/kg) \\
\hline Estación 1 & 973 \\
Estación 2 & 896 \\
Estación 3 & 917 \\
Estación 4 & 881 \\
Estación 5 & 980 \\
Estación 6 & 865 \\
Promedio & 918,67 \\
Límite permisible (RAOHE,2001) & 1000 \\
\hline Tabla 8 Contenidos de TPH en sedimento del ramal B Estero Salado \\
\end{tabular}

\section{Conclusiones.}

De las seis estaciones correspondientes al tramo B del Estero Salado se obtuvieron que en muestras líquidas la estación 6 (Puente Zic - Zac) tuvo los mayores contenidos de arsénico y plomo con 0,0090 y $0,0382 \mathrm{mg} / 1$ respectivamente, las mayores concentraciones de cadmio 
0,0015 mg/l y fenoles 0,025 mg/l estuvieron en la estación 4 (Centro Comercial Albán Borja) y en la estación 2 (Avenida Miraflores) se reportó el mayor contenido de mercurio 0,00262mg/l. Los hidrocarburos totales de petróleo en todas las estaciones tuvieron la misma concentración $0,04 \mathrm{mg} / \mathrm{l}$.

Mientras tanto en prototipos sedimentarios la estación 5 (Avenida Las Monjas) tuvo las mayores concentraciones de plomo $78 \mathrm{mg} / \mathrm{kg}$ e hidrocarburos totales de petróleo $980 \mathrm{mg} / \mathrm{kg}$, el cadmio $3,51 \mathrm{mg} / \mathrm{kg}$ y el mercurio $0,4 \mathrm{mg} / \mathrm{kg}$ sus contenidos mayores se presentaron en la estación 3 (Av. Dr. Ignacio Cuesta Garcés) y estación 1 (Complejo Polideportivo de Miraflores) respectivamente. El arsénico en todas las estaciones tuvieron la misma concentración 0,6 mg/kg.

Dentro del estudio se caracterizaron los siguientes parámetros:

Sólidos totales, $\mathrm{DBO}_{5}$, DQO y $\mathrm{pH}$, en la cual los sólidos totales se encuentran fuera del límite máximo permisible según la tabla 4a del reglamento ambiental de operaciones hidrocarburíferas del Ecuador (RAOHE). Este parámetro obtuvo un promedio en el tramo B de $3267 \mathrm{mg} / \mathrm{l}$ superando casi el doble de lo permitido $1700 \mathrm{mg} / \mathrm{l}$, este valor, se debe a la cantidad total de residuos sólidos filtrables (sales y residuos orgánicos) presentes en dicho sector. La $\mathrm{DBO}_{5}$ tuvo un promedio de 30,33 mg/l, en la cual está dentro del límite permitido $40 \mathrm{mg} / \mathrm{l}$, según lo establecido en la tabla 10 del RAOHE.

La DQO fue otro parámetro cuyas concentraciones estuvieron dentro del límite permitido $350 \mathrm{mg} / \mathrm{l}$ (Tabla 4a RAOHE) ya que sus contenidos se ubicaron en 49,67 mg/l, el potencial hidrógeno $(\mathrm{pH})$ sus unidades obtuvieron un promedio de 7,52 (ligeramente alcalino) que al igual 
de las anteriores cuantificaciones se encuentran dentro de lo permitido de acuerdo a la tabla 2 del TULA $6,5-9,5$.

Se elaboró un plan de remediación para mitigar contaminantes, con la técnica ex situ descontaminación físico química lavado de suelo, debido a que depura un porcentaje superior del $70 \%$ de contaminantes, a pesar de su alto costo son rápidas, consiguiendo una recuperación completa de la zona afectada. Cabe mencionar que no se puede realizar la remediación in situ en el sedimento marino del Estero Salado, por cuanto a las técnicas que se emplean podrían liberar el contenido de metales pesados e hidrocarburos debido al ascenso y descenso de la marea, dando el paso de los contaminantes a lo largo del Golfo de Guayaquil.

\section{Agradecimiento}

A la Maestría en Gestión Ambiental de la Facultad de Ingeniería Química de la Universidad de Guayaquil, por su formación académica en mí.

Al personal de campo y laboratorio del Grupo Químico Marcos, por su colaboración en la parte operativa de este trabajo de titulación.

A los ingenieros Delia Carrión León y José Hidalgo Torres de la Unidad de Titulación Especial (UTE), por sus aportes en el curso de titulación especial.

A mi tutora Ing. Judith Chalén Medina, Msc y revisora metodológica Ing. Mireya Bermeo Garay, Msc, por brindarme su apoyo y contribución en este proyecto de titulación.

A los ingenieros (as) Msc: Sandra Ronquillo, Mariana Navarro y Jaime Fierro, por formar parte del tribunal de sustentación. 


\section{Bibliografía.}

Alonso, R. (junio de 2012). Proyecto de recuperación de suelos contaminados por hidrocarburos. Obtenido de Universidad Autónoma de Barcelona: http://ddd.uab.cat/pub/trerecpro/2012

Aragón, C. (noviembre de 2013). Diseño a escala labotarorio y piloto de un sistema de remediación electrocinética de suelos contaminados por metales pesados. Obtenido de Escuela Politécnica Nacional: bibdigital.epn.edu.ec

Calero, M. (2010). La Gobernanza del Estero Salado. Obtenido de UTPL: dspace.utpl.edu.ec

Canadian Environmental Quality Guidelines. (2011). Directrices de calidad de sedimentos para la proteccion de la vida acuatica. Obtenido de Consejo Canadiense de ministros del medio ambiente: ceqgrcqe.ccme.ca/

Cando, M. (septiembre de 2011). Determinación y análisis de un proceso de biorremediación de suelos contaminados por hidrocarburos. Obtenido de Universidad Politécnica Salesiana: dspace.ups.edu.ec

Cardenas, M. (2010). Efecto de la contaminación hidrocarburífera sobre la estructura comunitaria de macroinvertebrados bentónicos presentes en el sedimento del Estero Salado. Obtenido de Universidad de Guayaquil: http://repositorio.ug.edu.ec/handle/redug/755

Cervantes, J. (1 de junio de 2012). Proyecto de recuperación del Estero Salado. Obtenido de blog: jeniffercervanteszambrano.blogspot.com

Diario El Telegráfo. (27 de agosto de 2012). La contanimación del agua: del estero al océano. La contanimación del agua: del estero al océano, pág. Seccion Columnista.

Guarco, K. (2015). Tratamiento del sedimento marino del ramal B del Estero Salado para la Remoción de

$$
\text { metales pesados. Obtenido de ESPOL: }
$$

http://www.dspace.espol.edu.ec/xmlui/handle/123456789/31957

Jimenez, D. (2012). Cuantificación de metales pesados (Cadmio, Cromo, Níquel y Plomo) en agua superficial, sedimentos y organismos (Crassostrea columbiensis) Ostión de Mangle en el Puente Portete el Estero Salado Guayaquil. . Obtenido de Universidad de Guayaquil: http://repositorio.ug.edu.ec/handle/redug/1683

Kuffo, A. (2013). Niveles de Cadmio, Cromo, Plomo, y su Bioacumulación por Mytella

Strigata delimitando la zona urbano-marginal en el Estero Salado de Guayaquil. Obtenido de Universidad de Guayaquil: http://repositorio.ug.edu.ec/handle/redug/3310

M.I. Municipalidad de Guayaquil y Consorcio Lahmeyer Cimentaciones. (2000). Estudios de Pre factibilidad, Factibilidad y selección de la mejor alternativa del Plan Integral de la Recuperación del Estero Salado (PIRES). Guayaquil.

Marrungo, L. (2011). Evaluación de la contaminación por metales pesados en la ciénaga Soledad y Bahpia de Cispatá, Cuenca del Bajo Sinú, Departamento de Córdova. Obtenido de Universidad de Córdova: www3.unicordoba.edu.co

Medina, F., \& Monserrate, B. (28 de noviembre de 2011). Estudio de condiciones físicas, química y biólogicas en la zona intermarial de dos estaciones del Estero Salado con diferente desarrollo humano. Obtenido de ESPOL: www.dspace.espol.edu.ec 
MIDUVI - MAE. (Junio de 2013). Ministerio de Desarrollo urbano y vivienda - Ministerio del Ambiente. Obtenido de Generación y restauración de áreas verdes para la ciudad de Guayaquil: Guayaquil Ecológico: www.ambiente.gob.ec/guayaquil-ecologico/

Ortíz, I., Sanz, J., Dorado, M., \& Villar, S. (2014). Técnicas de recuperación de suelos contaminados Informe de vigilancia tecnológica. Obtenido de Universidad de Alcalá Dirección de Universidades e Investigación: www.jaravalencia.com/docu/

Paéz, M., Soriano, R., Torres, L., \& Vasconez, N. (2014). Factores que Contaminan el Estero Salado de la Ciudad Guayaquil. Obtenido de Universidad Agraria Del Ecuador: es.slideshare.net

RAOHE. (13 de Febrero de 2001). Reglamento ambiental de actividades hidrocarburiferas. Obtenido de Ministerio de Recursos no renovables: www.hidrocarburos.gob.ec

Rodríguez, F. (2013). Cuantificación de cadmio, plomo y níquel en agua superficial, sedimento y organismo (Mytella guyanensis) en los puentes Portete y 5 de junio del Estero Salado Guayaquil. Obtenido de Universidad de Guayaquil: http://repositorio.ug.edu.ec/handle/redug/1682

Serrano, R. (2012). Alternativas de tratamiento para la remoción de lecho marino del Estero Salado. Obtenido de Universidad de Guayaquil.

STANDARD METHODS, 22 TH EDITION. (Agosto de 2012). Tablas actualizadas de métodos aprobados. En Standard Methods for the Examination of Water and Wastewater (pág. 724). American Public Health Association. Obtenido de https://www.standardmethods.org/

TULAS. (2015). Texto Unificado de Legislación Secundaria. En TULAS, Texto Unificado de Legislación Secundaria (pág. 14). Quito: Lexis.

Volker, T., \& Velasco, A. (2012). Tecnologías de remediación para suelos contaminados.

México: Talleres gráficos Jimenez Editores e Impresiones. 